\title{
CORRECTION
}

\section{ALTTO: wake-up call for setting up clinical trials}

Lisa Hutchinson

Nat. Rev. Clin. Oncol. 10, 121 (2013); doi:10.1038/nrclinonc.2013.20

In the version of this article initially published online and in print, the text indicated that the ALTTO study was led by Otto Metger-Filho, but this trial is a substudy of the larger ALTTO trial. The text should have referred to "the ALTTO substudy" within the article. The error has been corrected for the HTML and PDF versions of the article published online. 